\title{
Lobaplatin for the treatment of SK-MES-I lung squamous cell line in vitro and in vivo
}

\author{
This article was published in the following Dove Press journal: \\ OncoTargets and Therapy \\ II July 2016 \\ Number of times this article has been viewed
}

\author{
Hongming Zhang' \\ Runzhe Chen ${ }^{2}$ \\ Shaoxing Yang ${ }^{3}$ \\ Wenjing Liu $^{3}$ \\ $\mathrm{Ke} \mathrm{Li}^{4}$ \\ Haijun Zhang ${ }^{2}$ \\ Xiaoli Zhu' \\ Baoan Chen ${ }^{2}$ \\ 'Department of Pulmonary Medicine, \\ ${ }^{2}$ Department of Hematology and \\ Oncology, Zhongda Hospital, Medical \\ School, Southeast University, Nanjing, \\ Jiangsu Province, ${ }^{3}$ Department of \\ Pulmonary Oncology, The 307th \\ Hospital of Chinese People's \\ Liberation Army, Beijing, ${ }^{4}$ Department \\ of Medical Oncology, Jiangsu Cancer \\ Hospital, Nanjing, Jiangsu Province, \\ People's Republic of China
}

\begin{abstract}
Lung squamous cell carcinoma is the second-largest histological subtype of lung cancer, which is the leading cause of cancer-related death worldwide. Lobaplatin, one of the third-generation platinum compounds, has shown encouraging anticancer activity in a variety of tumors. The aim of this study was to determine the therapeutic efficacy of lobaplatin on p53-mutant lung squamous cancer cells SK-MES-1. In order to evaluate the antitumor effect of lobaplatin, several in vitro and in vivo analyses were carried out, including Cell Counting Kit-8 (CCK-8), fluorescence-activated cell sorter, Western blot, and terminal deoxynucleotidyl transferase dUTP nick end labeling. Findings showed that lobaplatin could inhibit cell proliferation and induce apoptosis of SK-MES-1 cells in vitro through both intrinsic and extrinsic apoptotic pathways in a time- and dose-dependent manner. In addition, lobaplatin could arrest cell cycle at S phase in SK-MES-1. Lobaplatin has obvious antitumor efficacy in human SK-MES-1 xenograft models; therefore, it seems to be a promising candidate in lung squamous cancer therapy.
\end{abstract}

Keywords: lobaplatin, lung squamous cell carcinoma, apoptosis, cell cycle arrest

\section{Introduction}

Lung cancer is the leading cause of cancer-related death all over the world. ${ }^{1}$ In 2015 , estimated 221,200 new cases (115,610 in men and 105,590 in women) of lung and bronchial cancers were diagnosed, and 158,040 deaths (86,380 in men and 71,660 in women) were estimated in the US. ${ }^{2}$ Non-small-cell lung cancer (NSCLC) accounts for $\sim 85 \%$ of lung cancer, in which lung squamous cell carcinoma (LSQCC), the secondlargest histological subtype, contributes to $\sim 30 \%{ }^{3}$ and causes $\sim 400,000$ deaths per year worldwide. ${ }^{4}$ Platinum-based chemotherapy is recommended as first-line treatment of patients with advanced NSCLC. Specific molecular-targeted therapies have also been developed for the treatment. Although significant progress has been made in NSCLC treatment in the past years, only $17.4 \%$ of all patients with lung cancer survived 5 years or more after diagnosis. ${ }^{5}$ The $p 53$ gene was the first tumor-suppressor gene identified. Lung squamous cancer has a $81 \%$ p53-specific mutation rate, ${ }^{4}$ which can upregulate genes that inhibit apoptosis or promote chemoresistance. ${ }^{6}$ As a most common regimen, gemcitabine/cisplatin is used for patients with squamous cell carcinoma, ${ }^{7}$ yet their clinical applications were limited by side effects and acquired resistance. Moreover, patients with LSQCC could not benefit from pemetrexed and targeted agents (such as gefitinib and bevacizumab), which are widely used in the treatment of lung adenocarcinoma. ${ }^{4}$ Thus, novel and effective chemotherapeutic agents are urgently needed to improve the overall survival for the patients with lung LSQCC.

Lobaplatin, the third-generation platinum compound whose molecular formula is $\mathrm{C}_{9} \mathrm{H}_{18} \mathrm{~N}_{2} \mathrm{O}_{3} \mathrm{Pt}$ (Figure 1), was approved for the treatment of chronic myelogenous 


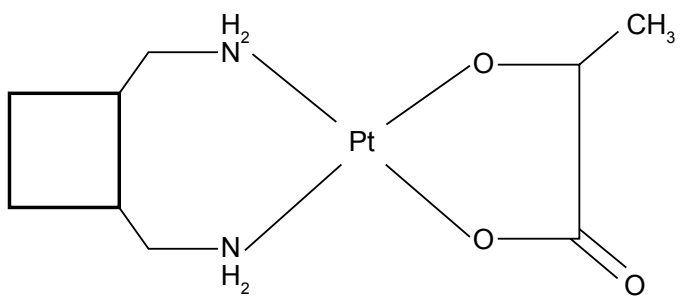

Figure I Chemical structure of lobaplatin

leukemia and inoperable metastatic breast and small-cell lung cancer in the People's Republic of China. ${ }^{8}$ With the minor side effects, incomplete cross resistance to cisplatin and stronger antitumor activity, it has shown great advantages over other chemotherapeutic agents in multiple tumors. ${ }^{9-20}$

There are few studies of lobaplatin on NSCLC up to date. Manegold et $\mathrm{al}^{21}$ studied the treatment efficacy of lobaplatin in 39 advanced NSCLC patients and only one of the 33 evaluable cases had a partial response (response rate $3 \%$ ). However, Xie et $\mathrm{al}^{22}$ found that the cytotoxicity of lobaplatin in vitro was similar to or higher than that of cisplatin and carboplatin in NSCLC cell lines, and it also showed significant antitumor activity in vivo. ${ }^{22}$ Furthermore, the combination of lobaplatin with paclitaxel could lead to more significantly enhanced activity compared with that of cisplatin combined with paclitaxel. ${ }^{22}$ An open-label, single group, multicenter, Phase II clinical trial found that the regimen composed of lobaplatin and navelbine was effective and well tolerated for Chinese patients with NSCLC with a response rate of $35.1 \%$ and a disease control rate of $78.4 \%{ }^{23}$

Since currently there are no studies on lobaplatin's therapeutic effects on lung squamous cell SK-MES-1, in this study, we investigate the therapeutic efficacy of lobaplatin on LSQCC in vitro and in vivo. Underlying molecular mechanisms were also explored to expand the clinical application of lobaplatin for LSQCC.

\section{Materials and methods}

\section{Compounds and reagents}

Lobaplatin was purchased from Hainan Changan International Pharmaceutical Co., Ltd. (Hainan, People's Republic of China). Minimum essential medium, minimum essential medium nonessential amino acid, sodium pyruvate, trypsin-EDTA, and penicillin/streptomycin were purchased from Thermo Fisher Scientific (Waltham, MA, USA). Dimethyl sulfoxide was obtained from Sigma Chemicals (Perth, Australia). Cell Counting Kit-8 (CCK-8) was purchased from Dojindo Molecular Technologies, Inc. (Kumamoto, Japan). Annexin V-FITC Apoptosis Detection Kit was purchased from BD (Franklin Lakes, NJ, USA). Antibodies to Bax and cyclin A2 were purchased from Proteintech Group Inc. (Chicago, IL, USA). Antibodies against Bcl-2, caspase-3, caspase-8, caspase-9, and poly ADP ribose polymerase (PARP) were purchased from Cell Signaling Technology, Inc. (Boston, MA, USA).

\section{Cell line and cell culture}

The human LSQCC cell line SK-MES-1 was provided by Nanjing Kebai Biotech. Co. Ltd (Nanjing, People's Republic of China). It was cultured in MEM medium supplemented with $10 \%(\mathrm{v} / \mathrm{v})$ heat-inactivated new-born calf serum (Thermo Fisher Scientific) with $1 \times 10^{5} \mathrm{U} / \mathrm{L}$ penicillin $\mathrm{G}$ and $1 \times 10^{5} \mathrm{U} / \mathrm{L}$ streptomycin in a humidified incubator at $37^{\circ} \mathrm{C}$ and $5 \% \mathrm{CO}_{2}$.

\section{Cell proliferation assay}

Cytotoxicity of lobaplatin to SK-MES-1 was examined by the CCK-8 assay. Cells were seeded in a 96-well culture plate at $4 \times 10^{3} /$ well. After overnight incubation, the cells were treated with $30 \mu \mathrm{M}, 10 \mu \mathrm{M}, 3.3 \mu \mathrm{M}, 1.1 \mu \mathrm{M}, 0.37 \mu \mathrm{M}, 0.12 \mu \mathrm{M}$, $0.04 \mu \mathrm{M}, 0.013 \mu \mathrm{M}, 0.0045 \mu \mathrm{M}, 0.0015 \mu \mathrm{M}$, and $0.0005 \mu \mathrm{M}$ lobaplatin for 24 hours, 48 hours, and 72 hours. A total of $10 \mu \mathrm{L}$ of CCK-8 solution was added to each well and incubated for additional 4 hours. The absorbance was measured at $450 \mathrm{~nm}$ wavelength using the ELX 800 Microplate Reader (BioTek Instruments, Inc, Winooski, VT, USA). Three wells were used for each concentration. The inhibitory rate of cell proliferation was calculated by the following formula:

$$
\text { Inhibition rate }(\mathrm{IR})=\left[1-\left(\frac{\mathrm{OD}_{\text {treated }}}{\mathrm{OD}_{\text {control }}}\right) \times 100 \%\right]
$$

The $50 \%$ inhibitory concentration was calculated by nonlinear regression fit of the mean values of the data obtained in triplicate independent experiments.

\section{Cell cycle distribution analysis}

Approximately $10^{4}$ SK-MES- 1 cells were harvested at room temperature after pretreatment with various lobaplatin concentrations $(0 \mu \mathrm{M}, 7.9 \mu \mathrm{M}$, and $15.8 \mu \mathrm{M})$ for 16 hours, 24 hours, and 32 hours. The supernatant was removed, and the cells were trypsinized, and then ice-colded $70 \%$ ethanol was added and fixed cells at $4^{\circ} \mathrm{C}$ overnight. After being washed and collected by centrifugation, cells were resuspended in phosphate-buffered saline (PBS) containing $50 \mu \mathrm{g} / \mathrm{mL}$ RNase and incubated at $37^{\circ} \mathrm{C}$ for 30 minutes. Then cells were stained in $100 \mu \mathrm{L}$ of $100 \mu \mathrm{g} / \mathrm{mL}$ propidium iodide (PI) and analyzed by flow cytometer (C6; BD). The cell cycle distribution was estimated according to the standard 
procedures. The distribution of cell cycle phases $\left(\mathrm{G}_{0} / \mathrm{G}_{1}, \mathrm{~S}\right.$, or $\mathrm{G}_{2} / \mathrm{M}$ phase) was calculated using Flowjo. All experiments were repeated and similar results were yielded.

\section{Apoptosis analysis}

SK-MES-1 cells were exposed to different concentrations of lobaplatin $(0 \mu \mathrm{M}, 15.8 \mu \mathrm{M}$, and $31.6 \mu \mathrm{M})$ for 48 hours and 72 hours. After that, $1 \times 10^{5}$ cells were trypsinized with EDTA-free trypsinogen, washed twice in PBS, and resuspended in $400 \mu \mathrm{L}$ of $1 \times$ binding buffer. A total of $5 \mu \mathrm{L}$ of annexin V-FITC and $5 \mu \mathrm{L}$ PI were added to the cells. After incubation at room temperature for 15 minutes in the dark, cells were then analyzed by a flow cytometer (C6; BD).

\section{Western blot}

Western blot was used to evaluate the expression level of the proteins of cell cycle and apoptosis-related signaling pathways, including cyclin A2, PARP, Bax, Bcl-2, caspase-3, caspase-8, and caspase-9. SK-MES-1 cells were exposed to various concentrations of lobaplatin for different periods of time, and then were washed twice with cold PBS and lysed in radioimmunoprecipitation assay buffer. The protein concentrations were measured using the bicinchoninic acid method. Total cell proteins were subjected to electrophoresis on $10 \%-12 \%$ polyacrylamide gels, transferred to nitrocellulose membranes, and probed with the following antibodies: cyclin A2, PARP, Bax, Bcl-2, caspase-3, caspase-8, and caspase-9 $(1: 1,000)$. The immunoblots were developed and visualized by ECL Western blot substrate (Thermo Fisher Scientific). Glyceraldehyde 3-phosphate dehydrogenase was used as internal control. The test of each group was repeated three times. The image $\mathrm{J}$ analysis system was used to measure the intensity of the bands.

\section{In vivo antitumor activity}

$\mathrm{BALB} / \mathrm{Ca}$ nu/nu male mice aged 5 weeks were purchased from Slac Laboratory Animal Center, Shanghai, the People's Republic of China. All the animal experiments were conducted under protocols approved by the animal ethics committee of the Medical School, Southeast University Human NSCLC xenograft of SK-MES-1 cells was established by inoculating viable SK-MES- 1 cells $\left(10^{7} / 100 \mu \mathrm{L}\right.$ PBS per mouse) into the right flank of male nude mice. When the average tumor volume reached $\sim 100 \mathrm{~mm}^{3}$, the mice were assigned randomly to control and treatment groups and treated with saline (d1, d8, and saline only, $\mathrm{n}=6)$ or lobaplatin $(\mathrm{d} 1, \mathrm{~d} 8$, $12 \mathrm{mg} / \mathrm{kg}, \mathrm{n}=6$ ) via tail vein, respectively. Tumor volume was calculated by the formula $v=\left(a \times b^{2}\right) / 2$, where " $a$ " is the longest diameter of the tumor, and " $b$ " is the diameter perpendicular to " $a$ ". Tumor size was measured every other day. The mice were sacrificed after 21 days, and the tumors were excised and stored at $-80^{\circ} \mathrm{C}$ until further analysis. The relative tumor volume (RTV) in individual mouse was determined as follows

$$
\mathrm{RTV}=\frac{V_{t}}{V_{0}}
$$

where $V_{t}$ is the volume on each measurement and $V_{0}$ is the volume on initial treatment. The therapeutic effect of a given compound was expressed in terms of $T / C(\%)$, which was calculated as

$$
\frac{T}{C}(\%)=\frac{\text { Mean RTV of the treated group }}{\text { Mean RTV of the control group }} \times 100 \%
$$

All experiments involving the mice were performed following the Guidelines for the Care and Use of Laboratory Animals of the National Institutes of Health.

\section{TUNEL analysis}

Formalin-fixed tumor tissues were embedded in paraffin and sectioned. Transferase dUTP nick end labeling (TUNEL) system (Hoffman-La Roche Ltd., Basel, Switzerland) was used to evaluate apoptosis in the tumor sections placed on slides according to the manufacturer's protocol. Tissue sections were analyzed to detect the localized green fluorescence of apoptotic cells and blue fluorescence of cell nuclei, and images were acquired and photographed using the Olympus IX51 fluorescence microscope (400×).

\section{Statistical analysis}

Statistical analysis was performed using SPSS19.0 software (IBM Corporation, Armonk, NY) package for Windows. Data were presented as the mean \pm SD. Statistical significance was calculated using a Student's $t$-test, with a probability level of $P<0.05$ being considered to be statistically significant.

\section{Results \\ Lobaplatin inhibited proliferation of SK-MES-I cells}

The CCK-8 assay was performed to evaluate the effects of lobaplatin on the proliferation of SK-MES-1 cells. The cells were treated with lobaplatin at the concentration of $30 \mu \mathrm{M}$, $10 \mu \mathrm{M}, 3.3 \mu \mathrm{M}, 1.1 \mu \mathrm{M}, 0.37 \mu \mathrm{M}, 0.12 \mu \mathrm{M}, 0.04 \mu \mathrm{M}$, $0.013 \mu \mathrm{M}, 0.0045 \mu \mathrm{M}, 0.0015 \mu \mathrm{M}$, and $0.0005 \mu \mathrm{M}$ for 48 hours and 72 hours, respectively. As shown in Figure 2, 


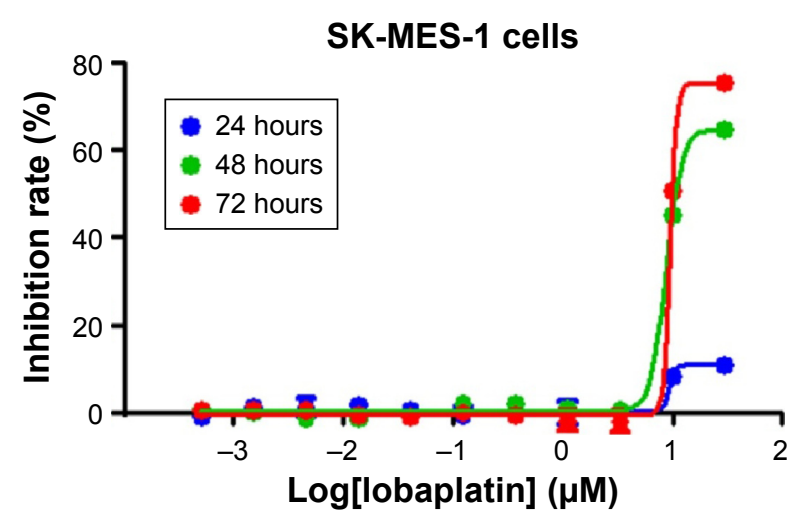

Figure 2 Effects of lobaplatin on the cell proliferation in SK-MES-I. Notes: Cells were exposed to various concentrations of lobaplatin $(0.0005-30 \mu \mathrm{M})$ for 24 hours, 48 hours, and 72 hours, followed by analysis with a CCK-8 assay. Abbreviation: CCK-8, Cell Counting Kit-8.

lobaplatin reduced the proliferation of SK-MES-1 cells in a time-dependent manner, and its 50\% inhibitory concentration is $15.8 \pm 1.09 \mu \mathrm{M}, 12.0 \pm 1.08 \mu \mathrm{M}$ for 48 hours and 72 hours, respectively. Thus, working concentrations were set to $7.9 \mu \mathrm{M}, 15.8 \mu \mathrm{M}$, and $31.6 \mu \mathrm{M}$ for subsequent experiments of apoptosis, cell cycle arrest, and Western blot.

\section{Lobaplatin triggered S cell cycle arrest}

Flow cytometry analysis after PI staining was used to detect the cell cycle arrest induced by lobaplatin. As displayed in Figure $3 \mathrm{~A}$ and $\mathrm{B}$, the percentages of SK-MES-1 in S phase after 32 hours of $7.9 \mu \mathrm{M}, 15.8 \mu \mathrm{M}$ lobaplatin treatment, and control group were $23.04 \% \pm 1.88 \%, 21.79 \% \pm 1.48 \%$, and $11.79 \% \pm 1.63 \%$, respectively. This suggested that $7.9 \mu \mathrm{M}$ and $15.8 \mu \mathrm{M}$ lobaplatin for 32 hours could induce $\mathrm{S}$ phase arrest significantly in SK-MES-1 $(P<0.05)$. A concurrent reduction of the cell population in $\mathrm{G} 1$ and $\mathrm{G} 2 / \mathrm{M}$ phases of the cell cycles was induced when SK-MES-1 was exposed to lobaplatin at the concentration of $7.9 \mu \mathrm{M}$ for 32 hours.

To further confirm the above results, the expression level of cyclin A2 was assessed. The expression level of cyclin A2 did not downregulate to basal level at the concentration of $7.9 \mu \mathrm{M}$ and $15.8 \mu \mathrm{M}$ of lobaplatin in 32 hours (Figure 3C). These results suggested that lobaplatin treatment $(7.9 \mu \mathrm{M}$ and $15.8 \mu \mathrm{M}$ ) induced $\mathrm{S}$ phase cell cycle arrest in SK-MES-1.

\section{Lobaplatin induced cell apoptosis in SK-MES-I cells}

Apoptosis was examined morphologically by DAPI staining. After treating with $15.8 \mu \mathrm{M}$ and $31.6 \mu \mathrm{M}$ of lobaplatin for 48 hours, the SK-MES-1 cells were stained with DAPI. Changes in cell nuclear morphology in lobaplatin-exposed cells were observed using a fluorescence microscope, which featured a marked increase in the quantity of apoptotic chromatin condensation and nuclear fragmentation depending on the concentration of lobaplatin. Despite that there were less cells to be stained due to higher lobaplatin concentration, chromatin condensation is more evident in panel $\mathrm{b}$ (31.6 $\mu \mathrm{M})$ compared with panel c (15.8 $\mu \mathrm{M}$; Figure 4A). Furthermore, annexin V-FITC/PI apoptosis detection kit was used to quantitatively determine whether lobaplatin induced SK-MES-1 cell apoptosis. After lobaplatin treatment for 48 hours and 72 hours, the apoptosis induction effects were observed (Figure 4B). The treatment groups showed more significant apoptosis compared with the control group $(P<0.05)$. The apoptosis rates were $55.2 \% \pm 1.1 \%$ and $67.1 \% \pm 1.5 \%$ when the SK-MES-1 cells were treated with $15.8 \mu \mathrm{M}$ and $31.6 \mu \mathrm{M}$ of lobaplatin for 48 hours, whereas they increased up to $72.1 \% \pm 1.5 \%$ and $81.4 \% \pm 0.8 \%$ when treated for 72 hours, respectively. Obviously, lobaplatin was able to induce apoptosis in a dosage- and time-dependent manner in SK-MES-1 (Figure 4C).

\section{Lobaplatin affected apoptosis-related proteins in SK-MES-I cells}

To further confirm the effect of lobaplatin-induced apoptosis in SK-MES-1, the expression level of some apoptosis-related proteins was evaluated by Western blot. As shown in Figure 5, lobaplatin increased the PARP level in a time-dependent manner. The expression of Bcl-2 significantly decreased while that of Bax increased in a concentration- and timedependent manner. The expression of cleavage-caspase-3, -8, and -9 increased. Cleavage-caspase- 8 increased in a concentration- and time-dependent manner. Nevertheless, cleavagecaspase- 3 and -9 increased in a time-dependent manner. The expression levels of PARP, caspase-8, and caspase-9 at the concentration of $31.6 \mu \mathrm{M}$ decreased compared with those at the concentration of $15.8 \mu \mathrm{M}$. Our results suggested that lobaplatin-induced apoptosis might adopt the mitochondrial apoptotic pathway and the extrinsic apoptotic pathway. Nonapoptotic cell death could be involved in inducing apoptosis by lobaplatin at higher concentration.

\section{Lobaplatin inhibited tumor growth and induces apoptosis in vivo}

To further investigate whether lobaplatin inhibits tumor growth in vivo, SK-MES-1 tumors were implanted in BALB/Ca nu/nu male mice. Our data showed that the lobaplatin-treated mice showed a slight but not significant body weight loss as compared with that in the control group (Figure 6A) and lobaplatin could cause inhibitory effects on the growth of SK-MES-1 


\section{A}

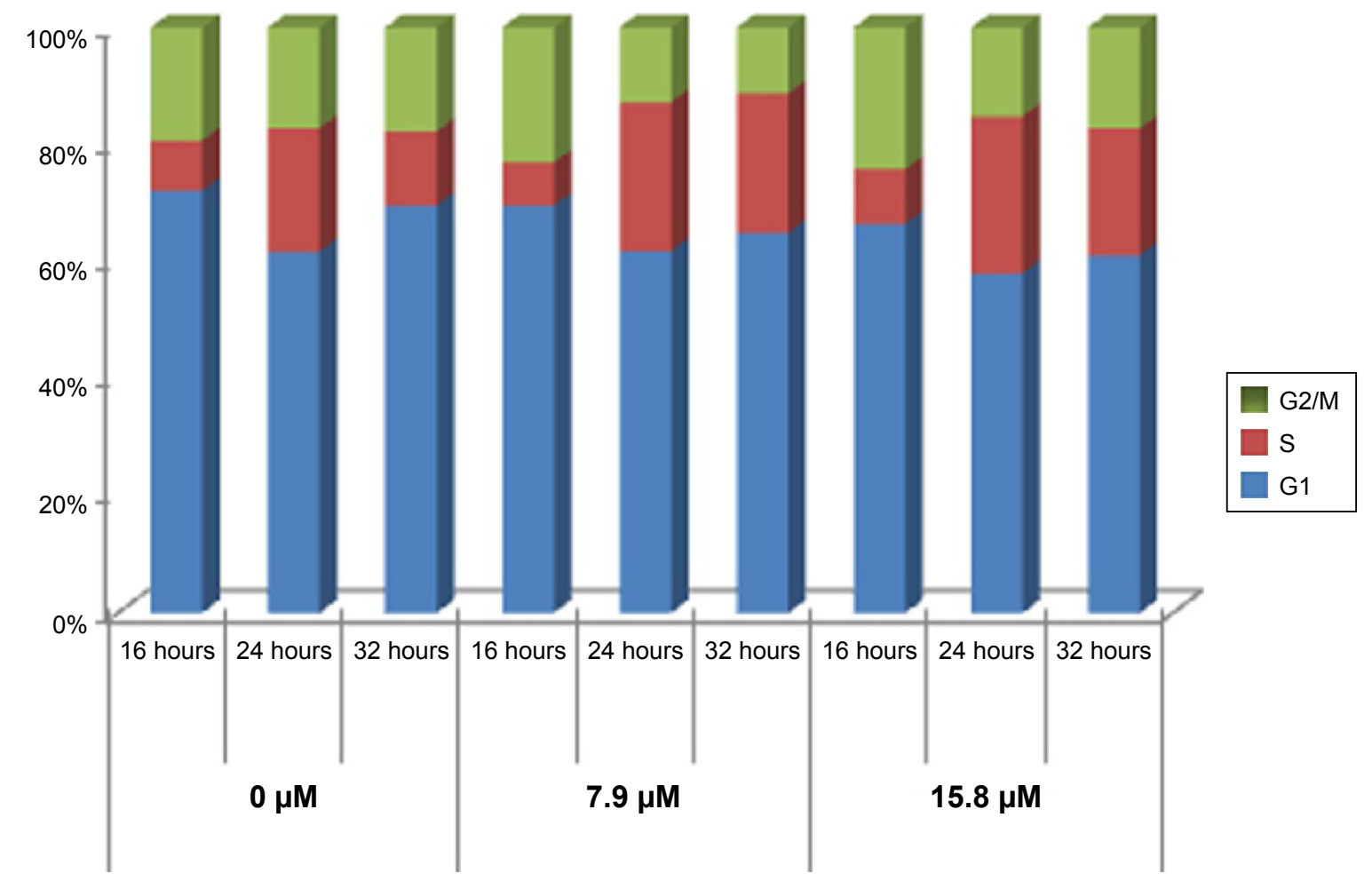

B
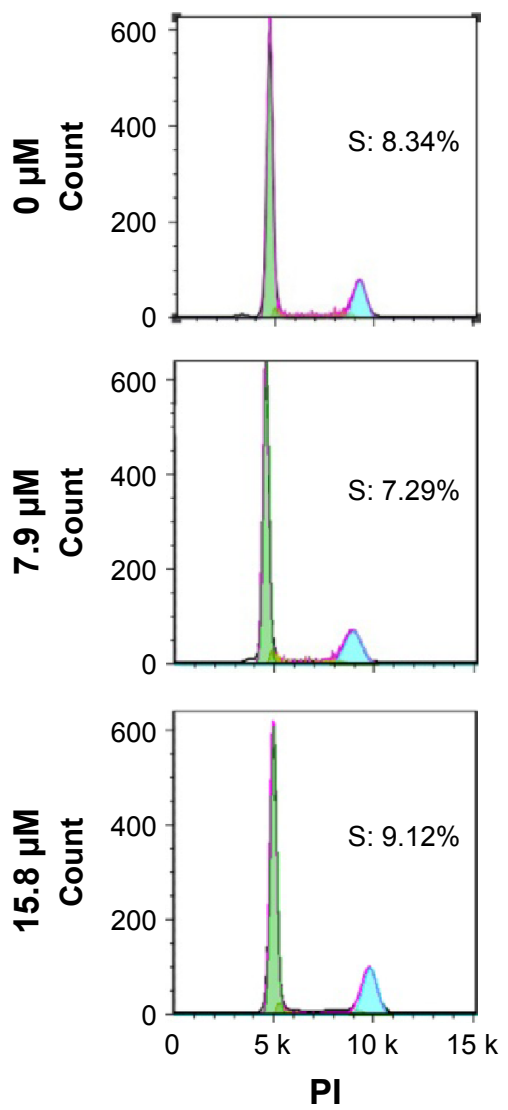

24 hours
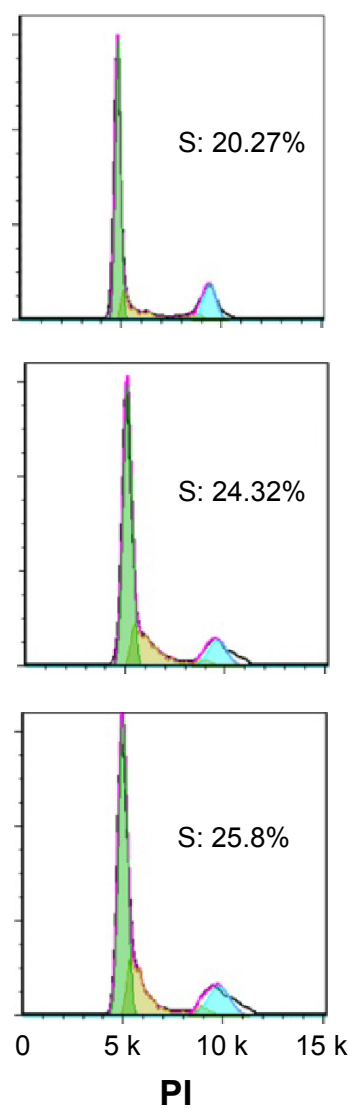

32 hours
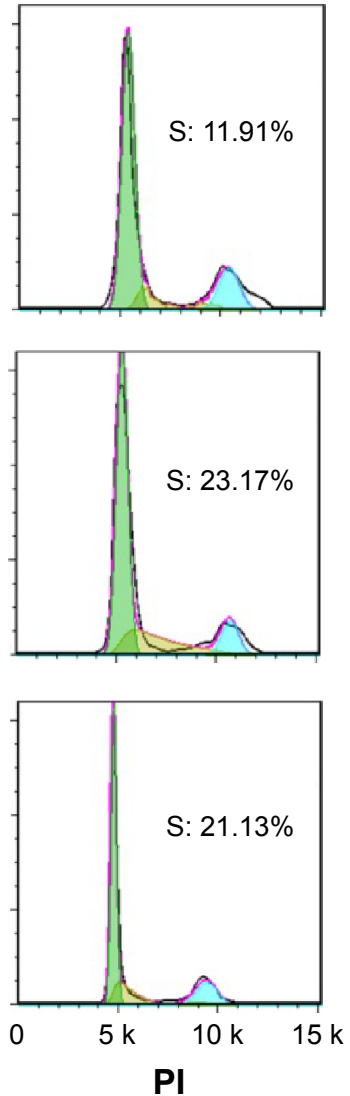

C
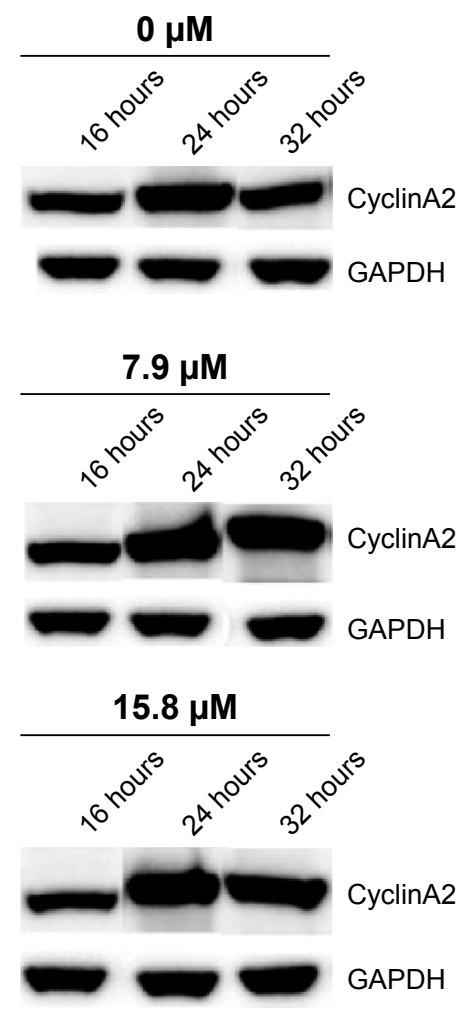

Figure 3 Effects of lobaplatin on cell cycle progression of SK-MES-I.

Notes: (A) Distribution of SK-MES-I cells at different phases of the cell cycle distribution. (B) Cells were exposed to various concentrations of lobaplatin ( $0 \mu$ M, $7.9 \mu$ M, and I5.8 $\mu \mathrm{M}$ ) for 16 hours, 24 hours, and 32 hours, followed by analysis of cell cycle by flow cytometry. (C) Western blot analysis of SK-MES-I cells treated (I6 hours, 24 hours, and 32 hours) with different concentrations $(0 \mu \mathrm{M}, 7.9 \mu \mathrm{M}$, and I5.8 $\mu \mathrm{M})$ of lobaplatin to evaluate the expression level of cyclinA2. 

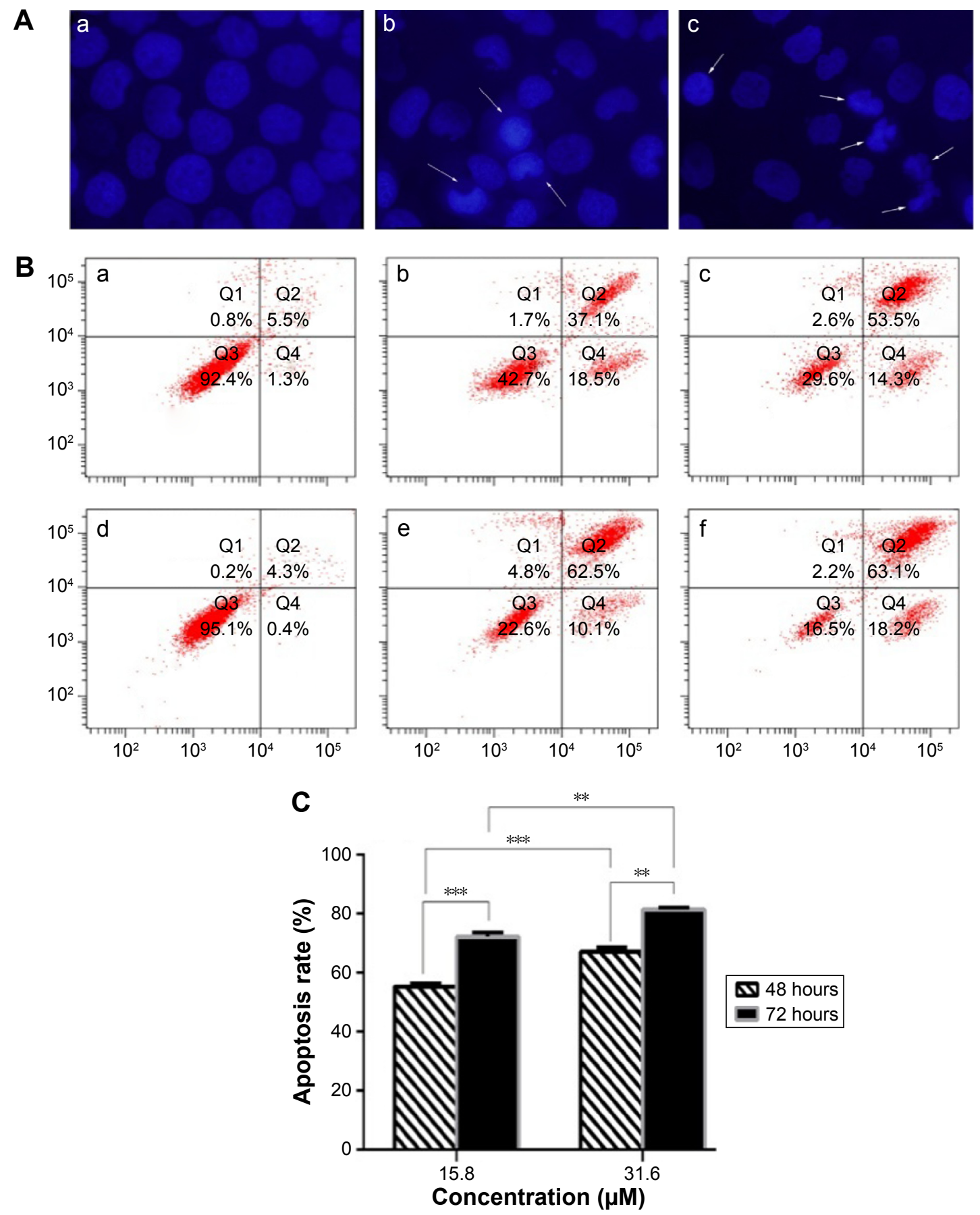

Figure 4 Induction of apoptosis in SK-MES-I cells by lobaplatin.

Notes: (A) Changes in cell nuclear morphology in lobaplatin-exposed cells were observed. SK-MES-I cells were treated with lobaplatin at $0 \mu M$ (a), I $5.8 \mu M$ (b), and 3 I. $6 \mu$ M (c) for 48 hours before staining with $2.5 \mu \mathrm{g} / \mathrm{mL}$ DAPI for 10 minutes and then analyzed using a fluorescence microscope (magnification, $\times 400$ ). The white arrows indicate chromatin condensation of nucleus. (B) SK-MES-I cells were treated with lobaplatin at indicated doses for 48 hours (b and c) and 72 hours (e and f), while a and $d$ work as controls. The apoptosis rates are calculated by adding up the percentage of Q2 and Q4, which appeared to be annexin V-positive. (C) The apoptosis rate was processed statistically. Data are expressed as mean $\pm S D$. $* * P<0.01$, $* * * P<0.001$.

tumors (Figure 6B-D). No regression was observed. In the control group, the mean tumor volume was $2,222 \pm 190 \mathrm{~mm}^{3}$, whereas that of the treatment group was $826 \pm 157 \mathrm{~mm}^{3}$ at the end of the experiment $(P<0.05)$. At the end of the study, the $T / C(\%)$ value was $45 \%$. The tumor inhibition rate was $63.9 \%$. These results demonstrated that the effect of lobaplatin was superior to that of the control group. However, we also found that the loss of body weight decreased after the two injections (Figure 6A). Samples were analyzed to detect the localized green fluorescence of apoptotic cells and blue fluorescence of cell nuclei using a fluorescence microscope. As shown in Figure 6E, lobaplatin could induce an enhanced apoptosis in 

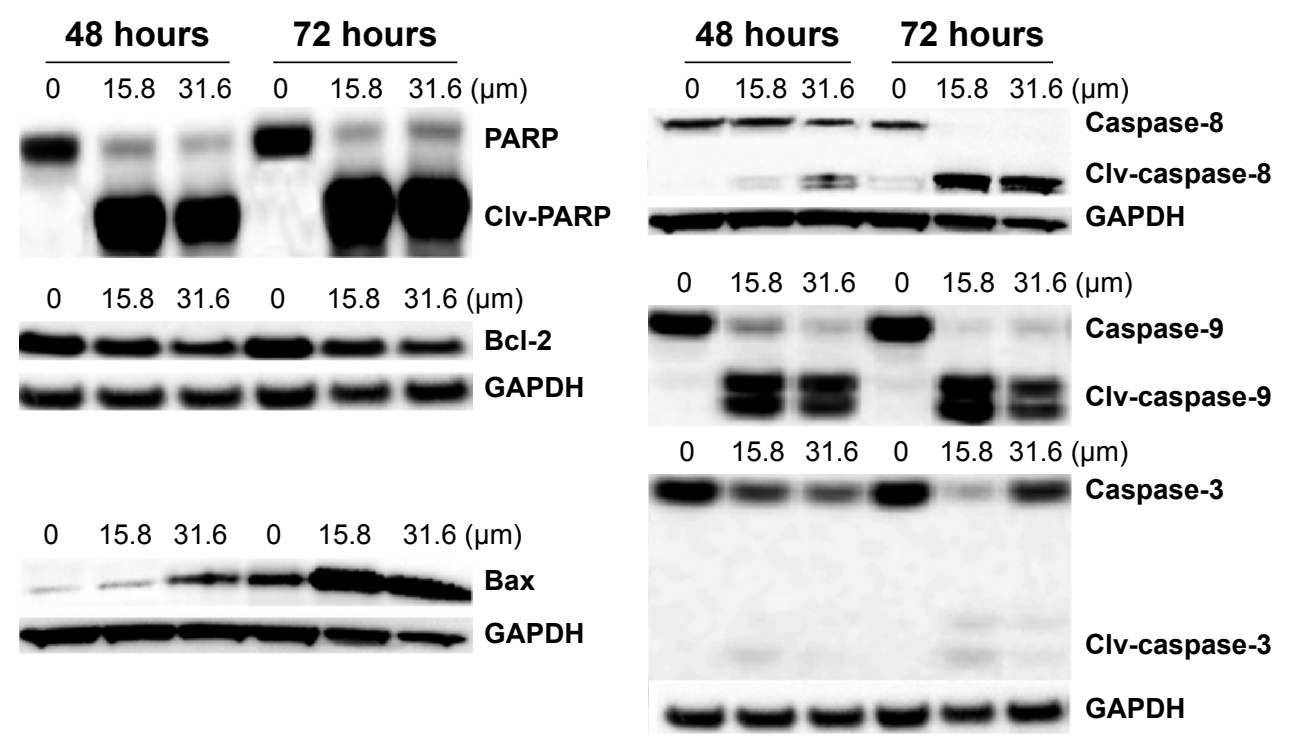

Figure 5 Western blot analysis of endogenous and lobaplatin-induced PARP, Bax, Bcl-2, caspase-3, caspase-8, caspase-9 protein levels in SK-MES-I.

Notes: K-MES-I cells were treated with different concentrations of lobaplatin at $0 \mu \mathrm{M}, 15.8 \mu \mathrm{M}$ and $31.6 \mu \mathrm{M}$ for 48 hours and 72 hours. Then, protein extracts were immunoblotted with specified antibodies against PARP, Bax, Bcl-2, caspase-3, caspase-8, and caspase-9. Activation levels of caspases and PARP (Clv bands) were determined.

The protein expression levels (relative to GAPDH) were assessed.

Abbreviations: GAPDH, glyceraldehyde 3-phosphate dehydrogenase; Clv, cleavage.

A

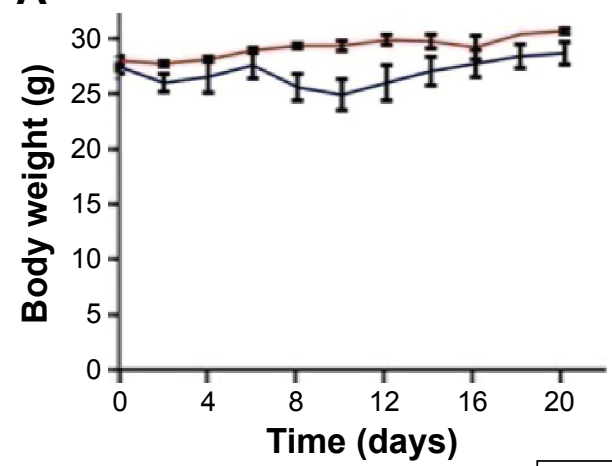

B

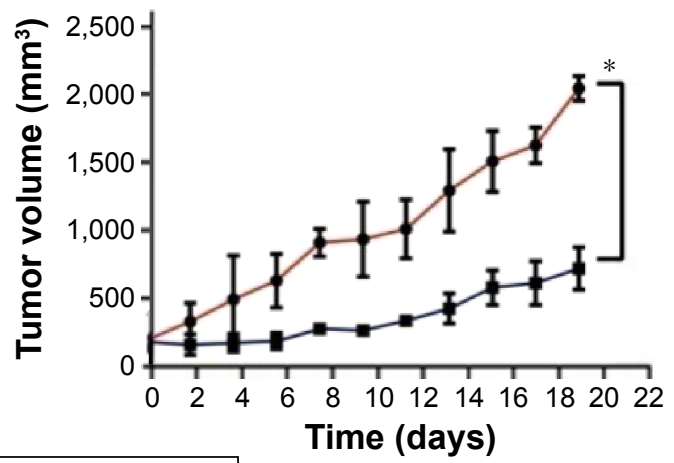

$\rightarrow-$ Control $\rightarrow=$ Lobaplatin

D

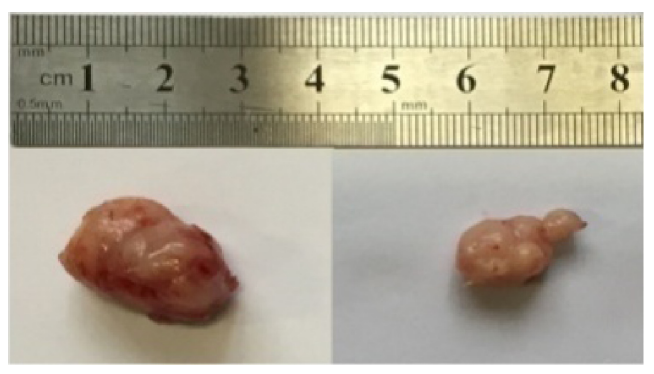

Control

Lobaplatin

Figure 6 (Continued) 


\section{$\mathbf{E}$} TUNEL positive cells (\%)

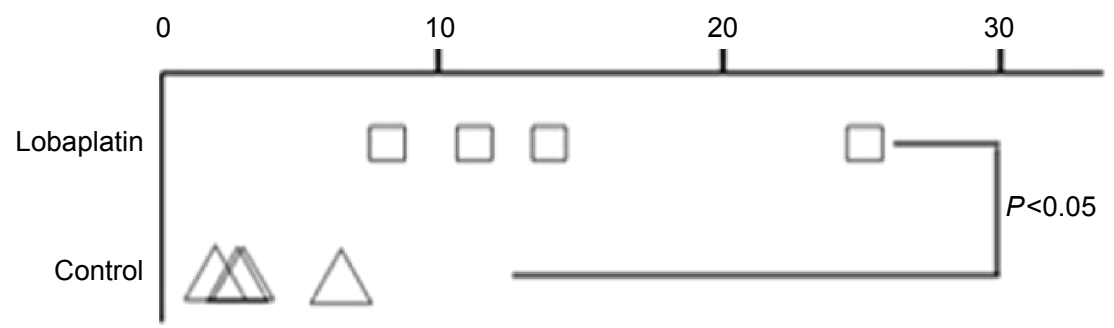

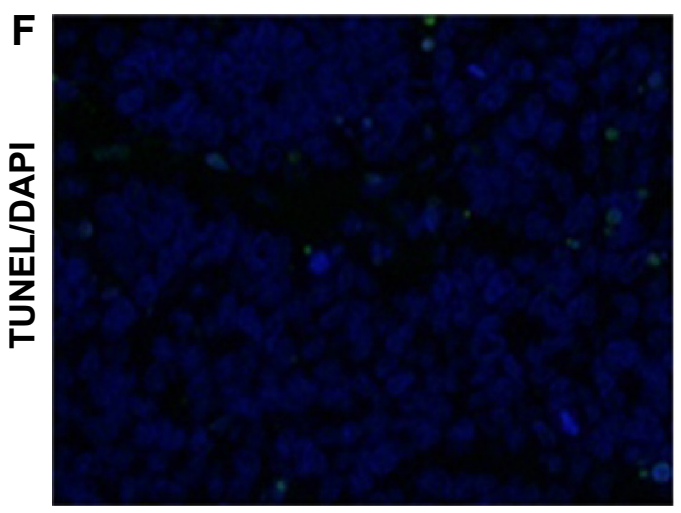

Control

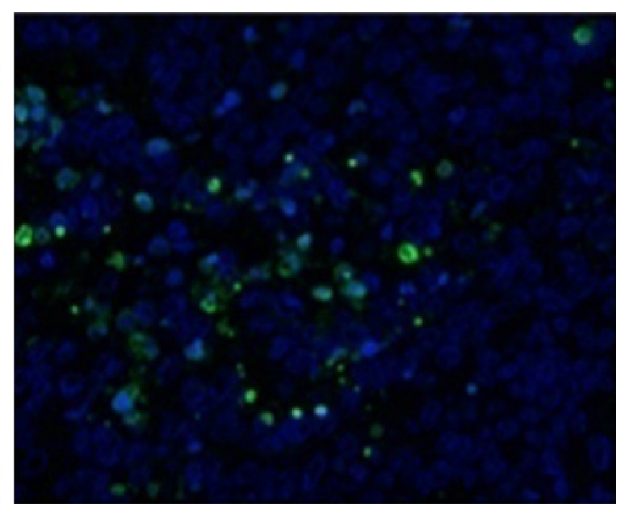

$12 \mathrm{mg} / \mathrm{kg}$ lobaplatin

Figure 6 The antitumor effect of lobaplatin on SK-MES-I human xenograft models.

Notes: (A) The average body weight of each group was expressed as mean \pm SD ( $n=6$ per group). (B) The mice transplanted with SK-MES-I xenograft were randomly divided into two groups and given injection of lobaplatin ( $12 \mathrm{mg} / \mathrm{kg}$ per week for a period of 2 weeks). The tumor volumes were expressed as mean \pm SD ( $\mathrm{n}=6 \mathrm{per}$ group). (C) Photos of mice with SK-MES-I xenograft tumors after 21 days of either treatment. (D) At the experimental end point, tumors were dissected and photographed. For C and D, only one mouse is demonstrated as an example for each group. (E) The TUNEL-positive rate of each tumor was analyzed. Magnification $\times 200$. (F) Representative photographs of TUNEL/DAPI staining of tumor tissues in different groups. $* P<0.05$.

Abbreviations: TUNEL, transferase dUTP nick end labeling; SD, standard deviation.

the tumor tissues in the treatment group. The percentage of apoptotic cells compared with the average number of cells per field was determined and graphically represented. The apoptotic rate in the treatment group increased significantly compared with that in the control group $(P<0.05)$. Taken together, lobaplatin has obvious antitumor efficacy in human SK-MES-1 xenograft models.

\section{Discussion}

Platinum-based chemotherapy is recommended as the firstline treatment of patients with advanced NSCLC. Cisplatin is the first-generation platinum compound and has become a major agent in the treatment of solid tumors, but its clinical application is limited by its side effects including gastrointestinal, renal, ototoxicity, and neurological toxicities. The main advantage of the carboplatin is myelosuppression, especially neutropenia that increases the risk of infection. Also, intrinsic or acquired resistance becomes the most prominent drawback to the use of these drugs. Lobaplatin, as the third generation of platinum antineoplastic agent, with its less side effects, incomplete cross-resistance to cisplatin, and stronger antitumor activity, has preliminarily shown its important role in cancer treatment. However, up to date, no studies on lobaplatin's therapeutic effects on LSQCC are available. Therefore, the present study investigated the effect of lobaplatin on human squamous cell line SK-MES-1 and the underlying molecular mechanisms to provide evidence for the further clinical application of lobaplatin in the therapy of LSQCC.

In this study, the CCK-8 assay indicated that lobaplatin exhibited evident cytotoxicity to SK-MES-1 in a timedependent manner.

Apoptosis, the programmed cell death, is one of the important antitumor mechanisms. A set of different apoptosis assays has been performed, including cytomorphological alterations, DNA fragmentation, detection of caspases, and cleaved substrates. ${ }^{24}$ The classic apoptotic morphologic changes include condensation of chromatin, nuclear fragmentation, and apoptotic bodies. In this study, apoptotic morphology was observed by DAPI staining after cells were treated by lobaplatin. It has been previously reported that lobaplatin induced apoptosis in human A549 lung adenocarcinoma cell line. ${ }^{22}$ Our results demonstrated that lobaplatin also induced apoptosis of SK-MES-1 cells in a dose- and time-dependent manner by fluorescence-activated cell sorter (FACS). 
Besides, in vivo TUNEL test has also proved that lobaplatin induced apoptosis of SK-MES-1 cells. Cisplatin can induce apoptosis in A549 cells by elevating the expression level of Bcl-2, Bax, PARP, and cleavage-caspase-3, -8 , and $-9 .{ }^{25}$ The proapoptotic protein Bax and antiapoptotic Bcl-2 were involved in the induction of intrinsic apoptosis. As a crucial apoptotic executive caspase, caspase- 3 is activated by proteolytic cleavage due to caspase- 8 and caspase- 9 , which are intrinsic and extrinsic apoptotic pathway initiators, respectively. Our results demonstrated that lobaplatin upregulated Bax, caspase-3, -8, and -9 and downregulated Bcl-2. Lobaplatin also elevated the expression level of cleaved PARP, which is mediated by the activation of executive caspases. However, at the concentration of $31.6 \mu \mathrm{M}$, the expression levels of PARP, caspase-9, and caspase- 3 decreased compared with the concentration of $15.8 \mu \mathrm{M}$, suggesting that most of the cells progressed to nonapoptotic cell death. This was indirectly substantiated by FACS analysis, where more cells were stained positive for both annexin-FITC and PI under 31.6 $\mu \mathrm{M}$ lobaplatin treatment. Our results suggested that intrinsic and extrinsic apoptotic pathways were involved in the induction apoptosis by lobaplatin in SK-MES-1.

Cell cycle arrest is also one of the mechanisms for cell growth inhibition induced by many anticancer drugs such as other platinum compounds: cisplatin $^{26}$ and carbolatin. ${ }^{27}$ Cisplatin treatment induced S-phase and G2/M-phase cell cycle arrest in A549 cells. ${ }^{26}$ Lobaplatin could arrest cells in the S phase in A549 cells. ${ }^{22}$ Human cyclin A2 begins to be degraded in early prometaphase and is completed at metaphase. ${ }^{28,29}$ Our results showed that the expression level of cyclin A2 is still upregulated until 32 hours but has declined to the basal level in the control group. These results confirmed that the SK-MES-1 cell cycle was arrested at S phase after being treated by lobaplatin.

Previous study has shown that lobaplatin has similar or better antitumor activity, and less toxicity, than cisplatin in human xenograft models such as A549 lung adenocarcinoma. ${ }^{22}$ Our study suggested that lobaplatin could reduce the tumor burden in the SK-MES-1 xenograft model. The results of the present study demonstrated that lobaplatin has obvious antitumor efficacy in human SK-MES-1 xenograft models. Considering the different toxicity profiles of platinum, lobaplatin might be a good option when cisplatin and carboplatin are not suitable.

\section{Conclusion}

Lobaplatin could significantly inhibit the growth of SKMES-1 cells in vitro and in vivo and induce cell apoptosis by upregulating the expression of Bax, PARP, caspase-3, -8, and -9 and downregulating the expression of Bcl-2. Moreover, lobaplatin could arrest cell cycle in S phase in SK-MES-1. Overall, our findings clearly concluded that lobaplatin is a promising candidate for the treatment of LSQCC.

\section{Acknowledgments}

This work was supported by the National Nature Science Foundation of the People's Republic of China (nos 81170492 and 81370673), the Key Medical Projects of Jiangsu Province (no BL2014078), the Key Discipline of Jiangsu Medicine (2011-2015), Wu Jieping Medical Foundation of Clinical Scientific Research Fund (no 320.6750.15069), and the Innovation Program for College Graduates of Jiangsu Province, People's Republic of China (no KYLX15-0186).

\section{Disclosure}

The authors report no conflicts of interest in this work.

\section{References}

1. Ferlay J, Soerjomataram I, Dikshit R, et al. Cancer incidence and mortality worldwide: sources, methods and major patterns in GLOBOCAN 2012. Int J Cancer. 2015;136(5):E359-E386.

2. Siegel RL, Miller KD, Jemal A. Cancer statistics, 2015. CA Cancer J Clin. 2015;65(1):5-29.

3. Hayashi T, Sano H, Egashira R, et al. Difference of morphology and immunophenotype between central and peripheral squamous cell carcinomas of the lung. Biomed Res Int. 2013;2013:157838.

4. The Cancer Genome Atlas Research Network. Comprehensive genomic characterization of squamous cell lung cancers. Nature. 2012; 489(7417):519-525.

5. Howlander N, Noone AM, Krapcho M, et al. SEER Cancer Statistics Review, 1975-2012. Based on November 2014, SEER Data Submission, [Posted to the SEER web site, April 2015]. Bethesda, MD: National Cancer Institute; 2015.

6. Freed-Pastor WA, Prives C. Mutant p53: one name, many proteins Genes Dev. 2012;26(12):1268-1286.

7. Scagliotti GV, Parikh P, von Pawel J, et al. Phase III study comparing cisplatin plus gemcitabine with cisplatin plus pemetrexed in chemotherapy-naive patients with advanced-stage non-small-cell lung cancer. J Clin Oncol. 2008;26(21):3543-3551.

8. State Food and Drug Administration Database. Available from: http:// app2.sfda.gov.cn/datasearchp/index1.do?tableId=25\&tableName= TABLE25\&scdw $=$ scdw\&Id=182332. Accessed 22 June 2016.

9. Harstrick A, Bokemeyer C, Scharnofkse M, Hapke G, Reile D, Schmoll HJ. Preclinical activity of a new platinum analogue, lobaplatin, in cisplatin-sensitive and -resistant human testicular, ovarian, and gastric carcinoma cell lines. Cancer Chemother Pharmacol. 1993;33(1):43-47.

10. Kavanagh JJ, Edwards CL, Freedman RS, et al. A trial of lobaplatin (D-19466) in platinum-resistant ovarian cancer. Gynecol Oncol. 1995; 58(1):106-109.

11. Yin CY, Lin XL, Tian L, Ye M, Yang XY, Xiao XY. Lobaplatin inhibits growth of gastric cancer cells by inducing apoptosis. World J Gastroenterol. 2014;20(46):17426-17433.

12. Wang Z, Tang X, Zhang Y, et al. Lobaplatin induces apoptosis and arrests cell cycle progression in human cholangiocarcinoma cell line RBE. Biomed Pharmacother. 2012;66(3):161-166.

13. Li X, Ran L, Fang W, Wang D. Lobaplatin arrests cell cycle progression, induces apoptosis and alters the proteome in human cervical cancer cell Line CaSki. Biomed Pharmacother. 2014;68(3):291-297. 
14. Wu Q, Qin SK, Teng FM, Chen CJ, Wang R. Lobaplatin arrests cell cycle progression in human hepatocellular carcinoma cells. J Hematol Oncol. 2010;3:43.

15. Degardin M, Armand JP, Chevallier B, et al. A clinical screening cooperative group phase II evaluation of lobaplatin (ASTA D-19466) in advanced head and neck cancer. Invest New Drugs. 1995;13(3): 253-255.

16. Yang F, Yu Y, Lei Q, et al. Lobaplatin arrests cell cycle progression, induces apoptosis and impairs migration and invasion in B16F10 melanoma cell line in vitro. Biomed Pharmacother. 2015;69: 402-408.

17. Dai HY, Liu L, Qin SK, He XM, Li SY. Lobaplatin suppresses proliferation and induces apoptosis in the human colorectal carcinoma cell Line LOVO in vitro. Biomed Pharmacother. 2011;65(3):137-141.

18. Meijer C, Mulder NH, Timmer-Bosscha H, Sluiter WJ, Meersma GJ, de Vries EG. Relationship of cellular glutathione to the cytotoxicity and resistance of seven platinum compounds. Cancer Res. 1992;52(24): 6885-6889.

19. Fiebig HH, Henb H, Vonpawel I, et al. Phase II clinical trial of lobaplatin (D-19466) in pretreated patients with small cell lung cancer. Onkologie. 1996;19:328-332.

20. Schmoll HJ, Kohne CH, Papageorgiou E, et al. Single agent lobaplatin is active in patients with esophageal squamous cell carcinoma: a phase II evaluation. Proc Am Soc Clin Oncol. 1995;14:483.

21. Manegold C, Drings P, Gatzemeier U, et al. Lobaplatin (D-19466) in patients with advanced non-small-cell lung cancer: a trail of the association for medical oncology (AIO) Phase II study group. Onkologie. 1996; $19: 248-251$
22. Xie CY, Xu YP, Jin W, Lou LG. Antitumor activity of lobaplatin alone or in combination with antitubulin agents in non-small-cell lung cancer. Anticancer Drugs. 2012;23(7):698-705.

23. Yang LQ, Yang Y, Qin SK, et al. Clinical study of lobaplatin combined with Navelbine for advanced non-small cell lung cancer. Chin Clin Oncol. 2006;11(12):890-894.

24. Elmore S. Apoptosis: a review of programmed cell death. Toxicol Pathol. 2007;35(4):495-516.

25. Wang LH, Li Y, Yang SN, et al. Gambogic acid synergistically potentiates cisplatin-induced apoptosis in non-small-cell lung cancer through suppressing NF-kappaB and MAPK/HO-1 signalling. $\mathrm{Br} J$ Cancer. 2014;110(2):341-352.

26. Suntharalingam K, Wilson JJ, Lin W, Lippard SJ. A dual-targeting, p53-independent, apoptosis-inducing platinum(II) anticancer complex, [Pt(BDI(QQ))]Cl. Metallomics. 2014;6(3):437-443.

27. Zhang Z, Zhang H, Hu Z, Wang P, Wan J, Li B. Synergy of 1,25dihydroxyvitamin D3 and carboplatin in growth suppression of SKOV-3 cells. Oncol Lett. 2014;8(3):1348-1354.

28. den Elzen N, Pines J. Cyclin A is destroyed in prometaphase and can delay chromosome alignment and anaphase. J Cell Biol. 2001;153(1): 121-136.

29. Geley S, Kramer E, Gieffers C, Gannon J, Peters JM, Hunt T. Anaphasepromoting complex/cyclosome-dependent proteolysis of human cyclin A starts at the beginning of mitosis and is not subject to the spindle assembly checkpoint. J Cell Biol. 2001;153(1):137-148.
OncoTargets and Therapy

\section{Publish your work in this journal}

OncoTargets and Therapy is an international, peer-reviewed, open access journal focusing on the pathological basis of all cancers, potential targets for therapy and treatment protocols employed to improve the management of cancer patients. The journal also focuses on the impact of management programs and new therapeutic agents and protocols on

\section{Dovepress}

patient perspectives such as quality of life, adherence and satisfaction The manuscript management system is completely online and includes a very quick and fair peer-review system, which is all easy to use. Visit http://www.dovepress.com/testimonials.php to read real quotes from published authors. 